\title{
Morbilidad dermatológica en pacientes atendidos por la Patrulla Aérea Civil Colombiana, 2008-2018
}

Dermatological morbidity in patients treated by the Colombian Civil Air Patrol, 2008-2018

\section{Nicolás Aguilar Medina ${ }^{1}$, Lucía Van Den Enden Medina², Felipe Jaramillo Ayerbe², Eduardo Carvajal De los Ríos 4}

1. Médico, residente en Dermatología, Universidad de Caldas, Manizales, Colombia

2. Médica, especialista en Dermatología, Universidad de Caldas. Docente, Programa de Dermatología, Universidad de Caldas, Manizales, Colombia

3. Médico, especialista en Dermatología, Universidad Nacional de Colombia. Dermatopatólogo, Universidad de New York. Jefe, Programa de Dermatología, Universidad de Caldas, Manizales, Colombia

4. Enfermero, especialista en Epidemiologia, Universidad El Bosque. Magíster en Economía de la Salud, Pontificia Universidad Javeriana, Bogotá, Colombia

\section{RESUMEN}

Introducción: la Patrulla Aérea Civil Colombiana es una organización privada sin ánimo de lucro, que durante aproximadamente 50 años ha brindado atención médica especializada, quirúrgica y de emergencia gratuita a personas en condiciones de pobreza y exclusión social o geográfica, por medio de brigadas mensuales en diferentes zonas rurales de Colombia. En sus archivos ha guardado información valiosa que es necesario conocer.

Materiales y métodos: se diseñó un estudio descriptivo, observacional, de corte transversal retrospectivo, que tiene como objetivo describir las características sociodemográficas, epidemiológicas y clínicas de los pacientes con patologías dermatológicas atendidos por la Patrulla Aérea Civil Colombiana durante los años 2008-2018.

Resultados: se realizaron 37 brigadas en 26 poblaciones de 14 departamentos de Colombia, donde se atendieron 2975 pacientes por dermatología, que tenían 3424 enfermedades. El 50\% de la población de estudio tenía 31 años o más. El 69\% eran mujeres y el 59,6\% eran mestizos. El acné fue la enfermedad más común, seguido de la pitiriasis versicolor, nevo melanocítico adquirido y dermatitis seborreica. Se encontraron 6 casos de lepra y 2 de lobomicosis.

Discusión: hay correlación en la frecuencia de enfermedades en las ciudades y en la muestra rural de nuestro estudio; sin embargo, el prurito sine materia se encontró concentrado en el Pacífico colombiano en mujeres recolectoras de “piangua”. Casi el 50\% de los carcinomas basocelulares encontrados se presentaron en dos poblaciones del Casanare.

Conclusiones: se recomiendan estudios para conocer la prevalencia de algunas infecciones tropicales en El Charco y La primavera. Se necesitan estudios de prevalencia de morbilidad dermatológica nacional con muestras poblacionales adecuadas.

PALABRAS CLAVE: enfermedades de la piel, Colombia, epidemiologia, acné vulgar, lobomicosis, lepra.
Correspondencia:

Nicolas Aguilar Medina

Email:

roberthcj@hotmail.com

Recibido: 21/02/19

Aceptado: 05/06/19

Conflictos de interés:

No se reportan conflictos de interés.

Financiación:

Ninguna. 


\section{SUMMARY}

Introduction: The Colombian Civil Air Patrol is a non-profit, private organization that, for almost 50 years, has provided specialized medical, surgical and emergency care for free to people in conditions of poverty and social or geographical exclusion, through monthly brigades in different rural areas of Colombia. On its archives, The Colombian Civil Air Patrol has kept valuable and must-know information.

Material and methods: A descriptive, observational retrospective cross-sectional study was designed, which objective was to describe the sociodemographic, epidemiological and clinical characteristics of patients with dermatological pathologies attended by The Colombian Civil Air Patrol during the years 2008-2018.

Results: 37 brigades were carried out in 26 populations of 14 departments of Colombia, where 2,975 patients with 3,424 diseases were attended by dermatologists. $50 \%$ of the study population was 31 years old or older. 69\% were women, and 59.6\% were mestizos. Acne was the most common disease, followed by pityriasis versicolor, acquired melanocytic nevus and seborrheic dermatitis. There were 6 cases of leprosy and 2 cases of lobomycosis.

Discussion: There is a correlation in the frequency of diseases in the cities and in the rural sample of our study. However, the pruritus sine materia was found concentrated in the Colombian Pacific in women gatherers of piangua. Almost 50\% of basal cell carcinomas were found in two Casanare populations.

Conclusions: Studies are recommended to know the prevalence of some tropical infections in El Charco and La Primavera. Studies on the prevalence of national dermatological morbidity with adequate population samples are needed.

KEY WORDS: Skin Diseases, Colombia, Epidemiology, Acne vulgaris, Lobomycosis, Leprosy.

\section{INTRODUCCIÓN}

La Patrulla Aérea Civil Colombiana (PAC) es una organización privada sin ánimo de lucro, que durante más de 50 años se ha dedicado a prestar atención médica, quirúrgica y de emergencia gratuita y sin trámites administrativos, a personas que viven en condiciones de pobreza, víctimas de alguna catástrofe o exclusión geográfica, en los lugares más apartados de Colombia. Realiza brigadas de salud mensuales y de tres días de duración, que requieren para su ejecución el transporte de aproximadamente dos toneladas de carga de equipos médicos, insumos quirúrgicos y medicamentos, además del voluntariado compuesto por 45 profesionales de la salud y pilotos. La PAC toma locaciones en la población elegida o el hospital de primer nivel, para transformarlo durante la brigada en un hospital de segundo nivel, que cuente con consultorios y salas de cirugía, lo que beneficia a cientos de personas con el desarrollo promedio de 1100 consultas médicas especializadas y 120 procedimientos quirúrgicos. Esto hace que la PAC esté en miras de convertirse en el hospital móvil de segundo nivel más importante de Colombia ${ }^{(1,2)}$.

El aumento gradual en la demanda de los servicios de salud en Colombia y en todos los países en general hace que sea necesario establecer estrategias o políticas en salud con miras a la reducción de la morbimortalidad, a la disminución de los costos en salud y a la inversión en la investigación. El conocer los motivos de consulta o la causa de enfermedad en una población permite realizar una planeación adecuada de la inversión de los recursos destinados a la salud ${ }^{(3)}$. La mejor forma de determinar la prevalencia real de las enfermedades dermatológicas es a través del examen físico cutáneo de una población general representativa. Si bien este es el modelo ideal para determinar tasas de prevalencia reales en un país o región, los costos que implica tal metodología limitan la realización rutinaria de este tipo de estudios en dermatología, hecho reflejado en la escasez de publicaciones que incluyan muestras poblacionales adecuadas en número y carentes de sesgos de selección ${ }^{(3,4)}$.

La PAC, a lo largo de los años que lleva de operación, ha guardado en sus archivos información demográfica y epidemiológica de muchas poblaciones colombianas, información valiosa que es necesario conocer. Aunque este estudio no puede establecer una prevalencia de enfermedades dermatológicas a nivel nacional, se convierte en una herramienta útil para tener una visión aproximada de las principales enfermedades de la piel en múltiples poblaciones rurales de distintas regiones de Colombia.

El objetivo principal de este estudio es describir las características sociodemográficas, epidemiológicas y clínicas de los pacientes con patologías dermatológicas atendidos por la PAC, durante los años 2008-2018. 


\section{MATERIALES Y MÉTODOS}

Se diseñó un estudio descriptivo, observacional, de corte transversal retrospectivo. La población está compuesta por todas las personas con enfermedades dermatológicas atendidas en las brigadas de salud realizadas por la PAC, durante el período comprendido entre el 2008 y el 2018, que fueron vistas por medicina general y, posteriormente, remitidas a evaluación por la especialidad de dermatología. Se incluyeron aquellos con diagnóstico clínico por el dermatólogo o confirmado con resultado de patología y se excluyeron los pacientes tratados por medicina general sin remisión y sin intervención por el especialista en dermatología. Los datos sociodemográficos y clínicos se tomaron de las historias clínicas en los archivos de la PAC, consolidado en un formato o sabana de Excel. Las edades se clasificaron en intervalos de 0-15 años, 16-45 años, 45-60 años y más de 61 años. Los diagnósticos se agruparon en cinco grupos de enfermedades: infecciosas, inflamatorias, neoplasias benignas, neoplasias ma- lignas y otros diagnósticos. El análisis se hizo en el programa estadístico Epidat 3.1. Se usaron estadísticos descriptivos (tendencia central y dispersión) para análisis observacional.

El comité de bioética de la Universidad de Caldas aprobó el estudio y lo consideró con riesgo mínimo, de acuerdo con la Resolución número 8430 de 1993 del Ministerio de Salud de la República de Colombia.

\section{RESULTADOS}

Los médicos generales de la PAC remitieron 3022 pacientes a dermatología. De estos, 2975 fueron finalmente evaluados por dermatólogo y 47 personas no asistieron a la consulta dermatológica especializada.

\section{Características sociodemográficas}

Las poblaciones y la cantidad de pacientes atendidos por brigadas se relacionan en la figura 1. Se

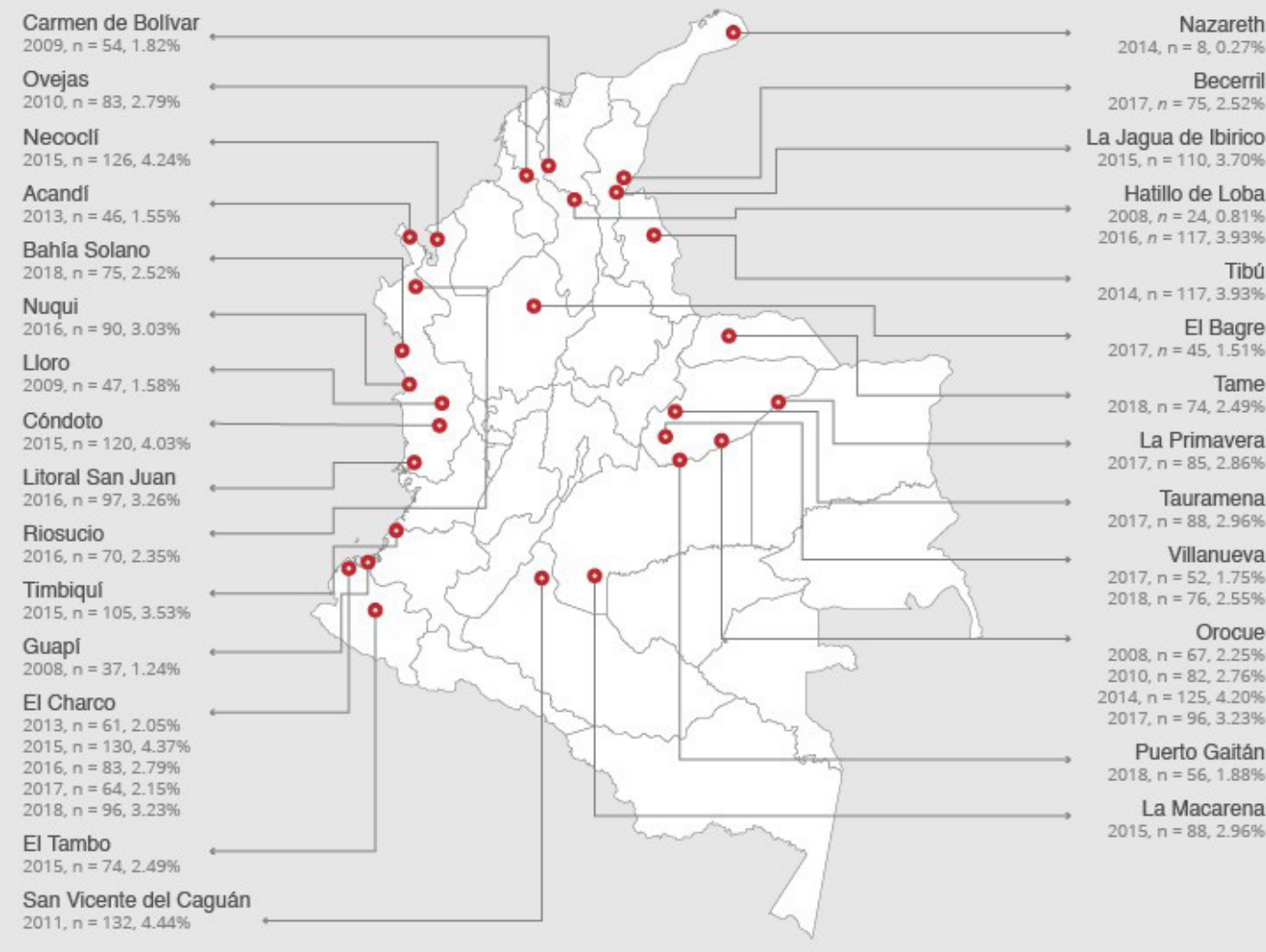

Figura 1. Poblaciones donde se realizaron brigadas de la PAC con atención dermatológica 2008-2018. 
realizaron 37 brigadas donde hubo atención por dermatología. Estas se hicieron en 26 poblaciones de 14 departamentos (Antioquia, Arauca, Bolívar, Caquetá, Casanare, Cauca, Cesar, Chocó, La Guajira, Meta, Nariño, Norte de Santander, Sucre y Vichada ), ubicados en cinco de las seis regiones naturales de Colombia (excepto la región insular).

En general, hubo una alta asistencia a la evaluación dermatológica, cercana al 100\% en casi todas las brigadas. Solo 47 inasistencias que se presentaron en dos poblaciones: en El Charco, Nariño, en 2015 no asistieron 22 pacientes y en 2016 no asistieron 8 pacientes; y en La Jagua de Ibirico, Cesar, en 2015 se reportaron 17 inasistencias.

Las características sociodemográficas como la edad, el sexo, la raza y la zona de procedencia se muestran en la tabla 1. El 50\% de la población de estudio tenía 31 años o más; el rango de edad más frecuente estuvo entre los 16 y 45 años (43,43\%), seguido de la población pediátrica, entre o y 15 años (27,45\%). El 69\% de los pacientes fueron del género femenino. El grupo racial predominante fue el mestizo, con el 59,66\%, seguido de afrocolombianos. en un 35,7\%, e indígenas, con un 4,64\%. La mayoría, correspondiente al 70,55\%, provenía del centro poblado donde se realizaron las brigadas y el 27,83\% de zonas dispersas rurales.

\section{Consolidado de diagnósticos}

De los 2975 pacientes evaluados por dermatología, 432 tenían un segundo diagnóstico y 17 un tercer diagnóstico, para un total de 3424 diagnósticos procesados en el estudio, que se agrupan en 164 enfermedades.

La distribución de las categorías diagnósticas se muestra en la figura 2. El 53,33\% corresponde a la categoría de enfermedades inflamatorias, siendo esta la más frecuente. $\mathrm{El} \mathrm{70 \%} \mathrm{de} \mathrm{los} \mathrm{diagnósticos} \mathrm{se} \mathrm{concen-}$ tran en 25 enfermedades, que se muestran en la tabla 2. El acné fue el principal motivo de consulta diagnosticado en 279 personas, lo que representa el 8,15\% del total de diagnósticos, seguido de la pitiriasis versicolor con el 4,96\%, nevo melanocítico adquirido con el

Tabla 1. Características sociodemográficas: edad, sexo, raza y zona

\begin{tabular}{|c|c|c|}
\hline \multicolumn{3}{|c|}{ Total, pacientes: 2975} \\
\hline Rangos de edad & $\mathrm{n}$ & $\%$ \\
\hline 0-15 años & 807 & $27,13 \%$ \\
\hline $16-45$ años & 1292 & $43,43 \%$ \\
\hline 46-6o años & 519 & $17,45 \%$ \\
\hline$>61$ años & 319 & $10,72 \%$ \\
\hline Sin datos & 38 & $1,28 \%$ \\
\hline \multicolumn{3}{|l|}{ Sexo } \\
\hline Femenino & 2053 & $69,00 \%$ \\
\hline Masculino & 922 & $31,00 \%$ \\
\hline \multicolumn{3}{|l|}{ Raza } \\
\hline Afrocolombianos & 1062 & $35,70 \%$ \\
\hline Indígena & 138 & $4,64 \%$ \\
\hline Mestizo & 1775 & $59,66 \%$ \\
\hline \multicolumn{3}{|l|}{ Zona } \\
\hline Centro poblado & 2099 & $70,55 \%$ \\
\hline Rural disperso & 828 & $27,83 \%$ \\
\hline Sin datos & 48 & $1,61 \%$ \\
\hline
\end{tabular}




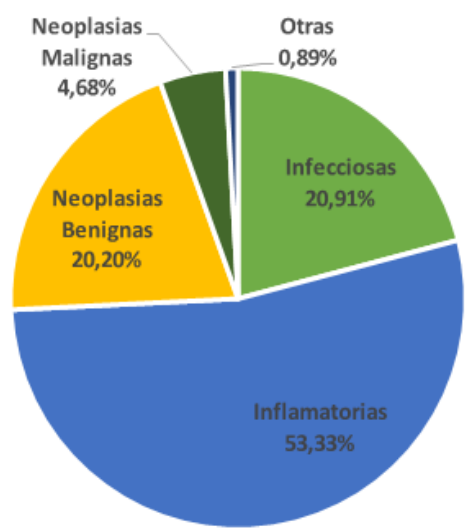

Figura 2. Distribución de las categorías diagnósticas.

Tabla 2. Principales 25 diagnósticos

\begin{tabular}{|c|c|c|}
\hline Diagnósticos & $n$ & $\%$ del total \\
\hline Acné & 279 & $8,15 \%$ \\
\hline Pitiriasis versicolor & 170 & $4,96 \%$ \\
\hline Nevo melanocítico adquirido & 161 & $4,70 \%$ \\
\hline Dermatitis seborreica & 142 & $4,15 \%$ \\
\hline Dermatitis de contacto & 140 & $4,09 \%$ \\
\hline Prurito sine materia & 134 & $3,91 \%$ \\
\hline Melasma & 115 & $3,36 \%$ \\
\hline Liquen simple crónico & 113 & $3,30 \%$ \\
\hline Verruga viral & 101 & $2,95 \%$ \\
\hline Prurigo estrófulo & 95 & $2,77 \%$ \\
\hline Acrocordón & 93 & $2,72 \%$ \\
\hline Dermatitis atópica & 86 & $2,51 \%$ \\
\hline Carcinoma basocelular & 76 & $2,22 \%$ \\
\hline Queratosis seborreicas & 75 & $2,19 \%$ \\
\hline Pitiriasis alba & 70 & $2,04 \%$ \\
\hline Miliaria & 64 & $1,87 \%$ \\
\hline Queloide & 63 & $1,84 \%$ \\
\hline Queratosis actínicas & 62 & $1,81 \%$ \\
\hline Quiste infundibular & 60 & $1,75 \%$ \\
\hline Tiña de los pies & 57 & $1,66 \%$ \\
\hline Foliculitis & 56 & $1,64 \%$ \\
\hline Vitíligo & 53 & $1,55 \%$ \\
\hline Psoriasis & 48 & $1,40 \%$ \\
\hline Tiña corporal & 48 & $1,40 \%$ \\
\hline Forunculosis & 42 & $1,23 \%$ \\
\hline Total & 2403 & $70,17 \%$ \\
\hline
\end{tabular}


4,70\%, dermatitis seborreica con el 4,15\%, dermatitis de contacto con el 4,09\%, prurito sine materia con el $3,91 \%$, melasma con el 3,36\%, liquen simple crónico con el 3,30\%, verruga viral con el 2,95\% y prurigo estrófulo con el $2,77 \%$. Los anteriormente descritos corresponden a los 10 principales diagnósticos.

\section{Enfermedades de consideración epidemiológica especial}

Se diagnosticaron 6 casos de leishmaniasis, cada uno en diferentes brigadas. Estos fueron: Acandí (Chocó)/2013, un caso proveniente de la población Media Luna; El Charco (Nariño)/2015, un caso proveniente de la población Playa Grande; La Jagua de Ibirico (Cesar)/2015, un caso; La Macarena (Meta)/2015, un caso proveniente de la población Lejanías; Puerto Gaitán (Meta)/2018, un caso; y San Vicente del Caguán (Caquetá)/2011, un caso proveniente de la población Carruya.

Se diagnosticaron 6 casos de lepra, cada uno en diferentes brigadas. Estos fueron: Becerril (Cesar)/2017, un caso; El Charco (Nariño)/2016, un caso; Orocué (Casanare)/2008, un caso; Villanueva (Casanare)/2017, un caso; y La Primavera (Vichada)/ 2017, un caso proveniente de la población El Rincón.

El carcinoma basocelular corresponde al decimotercer diagnóstico más frecuente, con 76 casos. Las brigadas realizadas en Villanueva y Orocué, ambas en el departamento del Casanare, fueron las que más diagnósticos hicieron, con 18 pacientes $(23,68 \%)$ y 15 pacientes $(19,74 \%)$, respectivamente.

Se encontraron 5 casos de carcinoma escamocelular, cada uno en diferentes poblaciones: Orocué (Casanare), Bahía Solano (Chocó), San Vicente del Caguán (Caquetá), El Charco (Nariño) y Nazareth (La Guajira).

\section{DISCUSIÓN}

Aunque el modelo del sistema de salud colombiano es reconocido por la Organización Mundial de la Salud como uno de los más solidarios del mundo ${ }^{(5)}$, hay zonas rurales y alejadas geográficamente en Colombia donde el acceso a los servicios sanitarios es limitado. En cuanto a dermatología respecta, se conoce que la distribución de los dermatólogos en el país es irregular y que se concentra en las grandes ciudades ${ }^{(6)}$; sumado al aumento gradual en la demanda de los servicios de salud en Colombia, muchas personas no cuentan con la oportunidad cercana de llegar a una evaluación dermatológica. La PAC ha contribuido en la atención en salud en muchas zonas alejadas del país, llevando recurso médico especializado a lugares y personas, que, de otra manera, no hubiesen recibido tal atención. Esto se ve reflejado en el gran aprovechamiento que las comunidades hacen al llegar una brigada de la PAC a su población, reflejada en la altísima asistencia a la evaluación dermatológica cercana al 100\% en cada una de las brigadas. En los últimos 10 años, solo 47 pacientes no asistieron a la evaluación dermatológica y 30 de estos casos se presentaron en el Charco ( $\mathrm{Na}-$ riño). Esta inasistencia en El Charco podría deberse a que es una población del Pacífico colombiano que no cuenta con caminería a través de la selva, ni existe transporte terrestre con las demás poblaciones cercanas, el transporte se efectúa por ríos y canales que son dependientes de corrientes y mareas ${ }^{(7)}$. Esto hace difícil e impredecible la movilidad de las personas de las poblaciones cercanas a El Charco para la asistencia a las brigadas.

Las mujeres son las que más consultan a dermatología en las brigadas de la PAC. Este comportamiento se correlaciona con los datos epidemiológicos de otros centros en ciudades capitales de Colombia: por ejemplo, en el consolidado 2016 del perfil epidemiológico del Hospital Universitario Centro Dermatológico "Federico Lleras Acosta”, en Bogotá, se muestra que, en la distribución de sexo, las mujeres representan el 63\% de los diagnosticados en esa entidad ${ }^{(8)}$. Esto podría atribuirse quizá a una mayor preocupación de las mujeres por su aspecto. Las personas jóvenes entre 16 y 45 años son las que más asisten a evaluación dermatológica, tanto en las zonas rurales donde la PAC realiza las brigadas como en los centros de ciudades capitales ${ }^{(4,8-10)}$.

Agrupadas por categorías, las enfermedades inflamatorias son las más frecuentes, tanto en las zonas rurales donde se hicieron las brigadas, como en el Hospital Universitario Centro Dermatológico "Federico Lleras Acosta”, en Bogotá, con un 53,3\% y un 45,4\%, respectivamente, del total de diagnósticos para cada uno ${ }^{(9)}$. El acné ha sido el diagnóstico más frecuente por varios años en el Hospital Universitario Centro Dermatológico “Federico Lleras Acosta”. Para el año 2012 represento el 17,9\% ${ }^{(9)}$ y para el año 2016, el 18\% ${ }^{(8)}$. En el Hospital Universitario San Vicente Fundación, en Medellín, el acné es el cuarto diagnóstico más común, con el $6,5 \%{ }^{(4)}$, datos similares a los encontrados en nuestro estudio, donde el acné es la entidad más frecuente. 
Otros diagnósticos que se encuentran entre los diez primeros realizados por los dermatólogos en las brigadas de la PAC y que se correlacionan con los principales de centros en ciudades capitales de Colombia son: dermatitis de contacto, dermatitis seborreica, melasma, verrugas virales y micosis superficiales $(4,8,9)$. Sin embargo, en la PAC hubo dos diagnósticos entre los principales que no se correlacionaron con los de las ciudades: prurito sine materia y prurigo estrófulo. El prurito sine materia fue la sexta entidad dermatológica más frecuente en la PAC; el 80\% de los casos se presentaron en brigadas realizadas en municipios del Pacífico colombiano (El Charco, Condoto, Nuquí, El Tambo y Litoral San Juan), siendo El Charco (Nariño) donde más casos se presentaron (34,33\%), particularmente en mujeres jóvenes entre los 16 y los 45 años $(84,2 \%)$, que desempeñaban labores de recolección de la Anadara tuberculosa (llamada vulgarmente "piangua” en Colombia y Costa Rica), un molusco que vive en zonas de pantano y manglar y que es un alimento típico de la Costa Pacífica Colombiana ${ }^{(11-13)}$. Podría existir algún agente etiológico desconocido de prurito en los ecosistemas de manglar y pantanos del Pacífico de Colombia. El prurigo estrófulo fue la décima condición dermatológica más frecuente en la PAC: el 92,3\% de los pacientes eran menores de 15 años, hallazgo que se correlaciona con las edades habitualmente reportadas en la literatura y en las zonas de clima tropical como Colombia ${ }^{(14,15)}$.

Teniendo en cuenta que las brigadas de la PAC se realizan en comunidades alejadas, que no son cabeceras departamentales, entre el año 2008 y 2018 la PAC ha confirmado solo 6 casos de leishmaniasis de los 110366 reportados al Sistema de Vigilancia en Salud Pública (SIVIGILA) y relacionados en el Boletín Epidemiológico del Instituto Nacional de Salud ${ }^{(16)}$. A la semana 33 de 2018, en Colombia se habían registrado 150 casos de lepra, siendo los departamentos del Valle del Cauca, Santander y Huila los de mayores notificaciones (27 casos, 15 casos y 12 casos, respectivamente) ${ }^{(17)}$. La PAC encontró 6 casos de lepra en seis diferentes brigadas, realizadas en departamentos que no corresponden a los de mayor notificación para el año 2018. Esto podría relacionarse con condiciones de desplazamiento y movilización poblacional, lo que da importancia al papel de la PAC en la detección de la enfermedad en territorios alejados del país.

En El Charco (Nariño), además de encontrarse 1 caso de lepra, se diagnosticaron 2 (2016 y 2018) de los 4 casos de esporotricosis (los otros 2 fueron en Orocué/2014 y Condoto/2015) y 2 (2018) de los 3 casos de cromomicosis (el otro en Orocué/2014). En el municipio de La Primavera (Vichada), se encontró 1 caso de lepra, además de 2 casos de lobomicosis (lacaziosis). Esta última enfermedad predomina en las áreas de alta pluviosidad, como el Amazonas y la cuenca del río Orinoco, donde está localizado el departamento del Vichada. Colombia ha reportado cerca de 50 casos de lobomicosis de los aproximadamente 150 que hay actualmente en la literatura $^{(18,19)}$.

El carcinoma basocelular es la neoplasia maligna dermatológica más frecuente encontrada por la PAC. Esto es igual a lo reportado por otros centros del país, en especial el Instituto Nacional de Cancerología, en donde también es la neoplasia maligna más común ${ }^{(20)}$. Llama la atención que en dos poblaciones del Casanare (Villanueva y Orocué) se concentran 33 de los 76 casos $(43,4 \%)$, datos que contrastan con el estudio de Sánchez y colaboradores ${ }^{(21)}$, donde se identificaron incidencias más altas de carcinoma basocelular en Bogotá D.C. y los departamentos de Antioquia, Cundinamarca y Boyacá.

Este es el primer estudio en Colombia con gran número de pacientes, que describe la morbilidad dermatológica con muestra de 26 poblaciones de 14 departamentos, en 5 de las 6 regiones naturales de Colombia, lo que proporciona una visión panorámica y aproximada de las principales enfermedades de la piel en múltiples poblaciones rurales del país.

Podemos concluir que, en las zonas rurales colombianas, hay necesidad de servicios médicos especializados, hecho que se ve reflejado en la altísima asistencia a las brigadas. Se recomiendan estudios en el Pacífico colombiano, para establecer algún agente etiológico de prurito, principalmente en El Charco (Nariño), donde, además, es necesario investigar la prevalencia de enfermedades de importancia epidemiológica como la leishmaniasis, la lepra, la esporotricosis y la cromomicosis. Adicionalmente, en La Primavera (Vichada), realizar estudios para lepra y lobomicosis. Es recomendable investigar la prevalencia de carcinoma basocelular en el Casanare (Villanueva y Orocué).

Es necesario implementar políticas de salud pública y herramientas tan valiosas como la teledermatología para la prevención y atención de las enfermedades dermatológicas más frecuentes en zonas rurales y dispersas de Colombia; además, la socialización y aplicación de las guías clínicas y de tratamiento que AsoColDerma ofrece en los niveles de atención primaria. Adicionalmente, es necesario el desarrollo de guías de 
otras enfermedades, como nevus y dermatitis seborreica, que son frecuentes según lo encontrado por el presente estudio.

Se recomiendan estudios de prevalencia de las enfermedades dermatológicas a nivel nacional, que incluyan muestras poblacionales adecuadas y carentes de sesgos de selección.

\section{AGRADECIMIENTOS}

Agradecimiento especial a la Patrulla aérea civil Colombiana por brindar la información y a la Dra. Angelica Vélez, por toda su colaboración en la recolección y organización de los datos.

\section{REFERENCIAS}

1. Patrulla Aérea Civil Colombiana [Internet]. Disponible en: www.patrullaaerea.org.

2. Patrulla Aérea Civil Colombiana [Internet]. Estrada Ocampo P. Brigada médico quirúrgica Puerto Gaitán-Meta, junio, 2018. Disponible en: https:// issuu.com/patrullaaereacivilcolombianao/docs/ informe_brigada_puerto_gaita_n.

3. Sanclemente G, Mahecha M, Guzmán C. Enfermedades de la piel más frecuentes en la consulta externa dermatológica del Hospital Universitario San Vicente de Paúl y del Hospital Infantil. Acta Med Colomb. 2001;26:240-44.

4. Sánchez G, Nova J, De la Hoz F. Factores de riesgo de carcinoma basocelular. Un estudio del Centro Nacional de Dermatología de Colombia. Actas Dermosifiliogr. 2012;103(4):294-300.

5. Melo L, Ramos JE. Borradores de economía. Algunos aspectos fiscales y financieros del sistema de salud en Colombia. Banco de la República. Número 624, 2010. Disponible en: http://www. banrep.gov.co/es/algunos-aspectos-fiscales-y-financieros-del-sistema-salud-colombia.

6. Ramírez A, Chalela JG, Ramírez J. ¿Cuántos dermatólogos hay en Colombia? Análisis de los datos de la Asociación Colombiana de Dermatología y Cirugía Dermatológica. Rev Asoc Colomb Dermatol. 2012;20(1):121-6.

7. Fundación Wikipedia, Inc. El Charco (Nariño). Disponible en: https://es.wikipedia.org/wiki/El_ Charco_(Nari\%C3\%B10).

8. Alcaldía de Marinilla-Antioquia. Consoidado 2016. Perfil epidemiológico del año 2016 de la población de Marinilla (eventos de interés en salud pública). Disponible en: http://www.marinilla-antioquia. gov.co/perfil-epidemiologico/consolidado-2016.

9. E.S.E Centro Dermatológico Federico Lleras [Internet]. Perfil epidemiológico, Consolidado 2012. Disponible en: http://www.dermatologia.gov.co/ index.php?idcategoria $=344 \&$ download $=\mathrm{y}$.

10. Castillo-Arenas E, Garrido V, Serrano-Ortega S. Skin conditions in primary care: an analysis of referral demand. Actas Dermosifiliogr. 2014;105(3):271-5.

11. El Tiempo. Silva M. La ruta de la piangua, el molusco del pacífico. Disponible en: https://www. eltiempo.com/archivo/documento/CMS-12748084.

12. Lucero C, Cantera J, Neira R. [The fisheries and growth of Ark Clams (Arcoida: Arcidae) Anadara tuberculosa in Málaga Bay, Colombian Pacific, 2005-2007. Rev Biol Trop. 2012;60(1):203-17.

13. Fundación Wikipedia, Inc. Anadara Tuberculosa. Disponible en: https://es.wikipedia.org/wiki/Anadara_tuberculosa.

14. Lozano AM, López JF, Zakzuk J, García E. Papular urticaria: A review of causal agents in Colombia. Biomedica. 2016;36(4):632-45.

15. Halpert E, Borrero E, Ibañez-Pinilla M, Chaparro P, Molina J, Torres M, García E. Prevalence of papular urticaria caused by flea bites and associated factors in children 1-6 years of age in Bogotá, D.C. World Allergy Organ J. 2017;10(1):36.

16. Instituto Nacional de Salud - Dirección de Vigilancia y Análisis del Riesgo en Salud Pública, Gobierno de Colombia. Boletín Epidemiológico Semanal (BES) 31-2018. Disponible en: https:// www.ins.gov.co/buscador-eventos/BoletinEpidemiologico/2018\%2oBolet $\% \mathrm{C}_{3} \% \mathrm{ADn} \% 20$ epidemiol $\% \mathrm{C}_{3} \% \mathrm{~B}_{3}$ gico $\% 20$ semana $\% 2031$.pdf.

17. Instituto Nacional de Salud - Dirección de Vigilancia y Análisis del Riesgo en Salud Pública, Gobierno de Colombia. Boletín Epidemiológico Semanal (BES) 34-2018. Disponible en: https:// www.ins.gov.co/buscador-eventos/BoletinEpidemiologico/2018\%20Bolet $\% \mathrm{C}_{3} \%$ ADn $\% 20$ epidemiol\%C3\%B3gico\%2osemana\%2034.pdf.

18. Riggioni VS, Díaz AS. Lacaziosis: micosis cutánea profunda, reporte de un caso. Rev Clin Esc Med. 2015;5(3).

19. Luna J, Villanueva J, Balcázar L. Lobomycosis: A case report. Dermatol Peru. 2012;22(2):111-4.

20. Pozzobon F, Acosta Á, Castillo J. Cáncer de piel en Colombia: cifras del Instituto Nacional de Cancerología. Rev Asoc Colomb Dermatol. 2018;26(1):12-7.

21. Sánchez G, Nova J, De la Hoz F, Castañeda C. Incidencia de cáncer de piel en Colombia, años 20032007. Piel. 2011;26(4):171-7. 